\title{
L'intégration des technologies numériques à l'évaluation des apprentissages à distance en enseignement supérieur : quelles transformations des pratiques évaluatives?
}

Digital technology integration in the context of distance learning assessment in higher education: What transformations are we seeing in assessment practices?

Incorporación de la tecnología digital a la evaluación del aprendizaje a distancia en la educación superior: cómo han cambiado las prácticas evaluativas?

https://doi.org/10.52358/mm.vi9.254

Julie Lyne Leroux, professeure

Université de Sherbrooke, Canada

julie.lyne.leroux@usherbrooke.ca

Jean-Marc Nolla, professeur

Université du Québec en Abitibi-Témiscamingue, Canada

jean-marc.nolla@uqat.ca 
RÉSUMÉ

Au Québec, des établissements d'enseignement supérieur au collégial, dont les programmes visent le développement de compétences, se tournent vers la formation à distance (FAD) et l'intégration des technologies numériques' (TN) pour accroitre l'accessibilité aux études. Sur la base des constats que les pratiques d'évaluation intégrant les TN sont peu documentées, le projet de recherche mené en amont à la pandémie vise l'identification de pratiques d'évaluation des apprentissages à distance mises en œuvre par des enseignants dans le cadre d'un cours d'un programme. La recherche qualitative/interprétative réalisée a permis d'apporter un éclairage au sujet des pratiques évaluatives intégrant les TN. Les résultats révèlent l'émergence de pratiques évaluatives qui intègrent les TN afin de répliquer, d'amplifier et de transformer l'évaluation des compétences.

Mots-clés : technologies numériques (TN), formation à distance (FAD), pratiques évaluatives, approche par compétences, enseignement supérieur

\section{ABSTRACT}

In Quebec, college-level higher education institutions, whose programs are aimed at competency development, are turning to distance learning (DL) and digital technology (DT) integration to expand the accessibility of studies. Based on the observation that assessment practices integrating DT are poorly documented, our research project undertaken prior to the pandemic seeks to identify $\mathrm{DL}$ assessment practices implemented by teachers in a program course. The qualitative/interpretive research performed has shed light on the assessment practices that make use of digital technologies. The results reveal the emergence of assessment practices that integrate DT in order to replicate, amplify, and transform competency assessment.

Keywords: digital technology (DT), distance learning (DL), assessment practices, competency-based approach, higher education

RESUMEN

En Quebec, las instituciones de enseñanza superior terciaria, cuyos programas tienen como fin el desarrollo de competencias, están volcándose hacia la educación a distancia (EAD) y a la incorporación de la tecnología digital (TD) para facilitar el acceso a los estudios. Basándose en que las prácticas de evaluación que incorporan TD están poco documentadas, el proyecto de investigación realizado antes de la pandemia tiene por objeto identificar aquellas prácticas

\footnotetext{
${ }^{1}$ Nous définissons les technologies numériques (TN) comme suit: "Technologies de l'information et des communications qui sont intégrées et utilisées dans l'ensemble des fonctions et des services [d'un établissement d'enseignement supérieur] pour recueillir, stocker, analyser et communiquer de l'information sous un format numérique avec [les différents groupes d'acteurs] » (Gouvernement du Québec, 2018, p. 84).
} 
de evaluación del aprendizaje a distancia utilizadas por los profesores en el marco de una de las materias de una carrera. La investigación cualitativa/interpretativa realizada permitió esclarecer los métodos evaluativos que incorporan TD. Los resultados revelan el surgimiento de prácticas de evaluación que utilizan dicha tecnología para reproducir, ampliar y transformar la evaluación de competencias.

Palabras clave: tecnología digital (TD), educación a distancia (EAD), prácticas de evaluación, aprendizaje basado en competencias, educación superior

\section{Introduction}

L'intégration des technologies numériques (TN) se répand à l'échelle mondiale et suscite une variété de pratiques en enseignement supérieur (Allen et Seaman, 2013; Gouvernement du Québec, 2015; Johnson, 2020; Johnson, Seaman, et Valetsianos, (2020), Seaman, Allen et Seaman, 2018; UNESCO, 2011). Les TN sont un incontournable de la formation à distance (FAD) dont l'intégration s'est grandement accrue au cours des deux dernières décennies et qui s'est accélérée au cours de la récente pandémie mondiale (COVID-19) (Detroz, Tessaro et Younès, 2020). La FAD est synonyme de formation hybride ou de formation en ligne (Audet, 2011; Gouvernement du Québec, 2015).

En plus de favoriser le déploiement de l'offre de formation à distance (FAD), l'accès facilité aux TN contribue à l'enrichissement de la qualité de la formation, soit de l'enseignement et de l'apprentissage (Audet, 2011; Lebrun, 2015; Papi, 2016; Papi, Gérin-Lajoie et Hébert, 2020). Les modalités de rétroaction et de régulation des apprentissages ont évolué : les rétroactions sont nombreuses, offertes dans des formats variés (écrites, audio, vidéo) et fournies aux étudiants plus rapidement. Alors que les TN entrainent des changements en enseignement supérieur, de nombreux défis sont soulevés (Burton, Blais et Gilles, 2013; Lebrun, 2015, Nizet, Leroux, Deaudelin, Béland et Goulet, 2016). Par exemple, quelles ressources technologiques doit-on retenir? Comment combiner les ressources technologiques aux modalités d'évaluation? Comment assurer la confidentialité et l'authentification des données recueillies? Dans le cadre de programmes qui accordent une place centrale au développement de compétences, les pratiques d'évaluation des apprentissages à distance au collégial, premier palier de l'enseignement supérieur au Québec, sont peu documentées. Bien que des études ont apporté un éclairage au sujet des pratiques d'évaluation des apprentissages dans un programme en approche par compétences (Bélanger et al., 2012; Leduc, Raîche et Blais, 2012; Leroux, 2010), les études qui portent un éclairage sur les pratiques d'évaluation des apprentissages à distance d'enseignants du collégial sont rares. Par exemple, les travaux de Facchin (2018) portent essentiellement sur l'amélioration des pratiques d'évaluation formatives en général et sur la rétroaction en particulier de tuteurs qui interviennent en FAD.

Dans l'intention de comprendre les pratiques évaluatives des apprentissages à distance d'enseignants en enseignement supérieur au collégial dans le cadre d'un cours d'un programme en approche par compétences (APC), nous commencerons par situer la problématique à laquelle répond la recherche. Nous présenterons ensuite le cadre de référence et le cadre méthodologique afin d'identifier des pratiques d'évaluation formative et sommative/certificative des apprentissages à distance. Enfin, nous conclurons par la formulation d'enjeux liés à la mise en œuvre de pratiques d'évaluation des apprentissages à distance en enseignement supérieur. 


\section{Des pratiques d'évaluation des apprentissages à distance en émergence}

Au Québec, les programmes réformés accordent une place centrale au développement de compétences dans les établissements d'enseignement au collégial. Or, depuis la mise en œuvre de l'approche par compétences, des enseignants rencontrent des difficultés à mobiliser les cadres théoriques et méthodologiques reliés à l'instrumentation objective et rigoureuse des évaluations (Tardif, 2006). D'autres soulignent également des difficultés pour développer des pratiques de jugement rigoureuses et valides dans un contexte où beaucoup manquent souvent de formation en évaluation (Leroux, 2016). De plus, les pratiques d'évaluation des enseignants sont parfois assujetties à des normes collectives peu ou mal adaptées à la FAD en enseignement supérieur (Nizet et al., 2016). En ce sens, les enseignants soulignent des défis par rapport à de la certification qui exige des ajustements et des appropriations en amont. Mais alors que les enseignants doivent évaluer les compétences des étudiants en tenant compte de ces défis en présentiel, ils ont également à composer avec des initiatives de FAD qui sont mises en œuvre dans plusieurs établissements d'enseignement supérieur sans une structuration et un fil conducteur sur le plan organisationnel (Gouvernement du Québec, 2015). Pourtant, les changements entrainés par un environnement technologique en constante évolution révèlent de nouvelles attentes. Les résultats de recherches montrent qu'une intégration réussie des TN dans le but de soutenir l'enseignement et l'apprentissage nécessite le développement d'une compétence numérique (Barrette, 2009; Gouvernement du Québec, 2018; 2019; Roy, Gruslin et Poelhuber, 2020; Tremblay, 2020).

En enseignement supérieur également, le développement de la compétence numérique qui s'inscrit dans le prolongement du plan d'action numérique (Gouvernement du Québec, 2018) tient compte de plusieurs exigences et de plusieurs défis liés à l'évaluation des compétences dans les différents programmes préuniversitaires et techniques au collégial. On relève le manque de formation des enseignants en évaluation des apprentissages, la sécurité et la crédibilité des évaluations, l'adaptation des pratiques et des politiques évaluatives, la disponibilité des ressources et l'obsolescence accélérée des outils à intégrer en évaluation. Mais si le cadre de l'évaluation en FAD comporte des alourdissements tant pour les étudiants que pour les enseignants, plusieurs travaux soulignent aussi des irritants associés à un nouveau rapport au temps (accélération) (Da Costa Cabral, Gremion, et Roblez, 2020; Gremion, 2018; Lakhal, Leroux et Martel, 2015; Nizet et al., 2016; Petit, 2016).

Mais alors que l'intégration des TN ne cesse de se complexifier et de contribuer à transformation des pratiques évaluatives, sur le terrain, dans des programmes de la formation régulière et continue, de nombreuses pratiques sont expérimentées par des enseignants (Bélec et Richard, 2019; Bélair, 2016; De Grâce, 2016). La transformation des pratiques évaluatives révèle, par exemple, la combinaison de nouvelles modalités d'enseignement et d'apprentissage (présentiel enrichi, hybride et tout à distance), d'une diversité de technologies numériques (les outils du Web 2.0 comme les tags, l'infonuagique, les blogues, les wikis, le balado) et des modalités d'interaction (étudiant-contenu, étudiant-enseignant, étudiant-étudiant). Toutefois, en fonction de l'intention d'évaluation (formative, sommative/certificative), les types de combinaisons restent à élucider.

Malgré la nécessité de prendre en compte cette nouvelle réalité technologique dans les pratiques des enseignants, une lacune à combler demeure : les pratiques d'évaluation des apprentissages à distance des enseignants du collégial sont peu documentées. Aucune étude récente, à notre connaissance, n'a investigué les pratiques d'évaluation des apprentissages à distance, que nous définissons pour la présente recherche comme les pratiques évaluatives mises en œuvre par des enseignants du collégial, qui utilisent 
les TIC et des outils du Web, pour évaluer les apprentissages des étudiants dans le cadre de formation à distance dans un programme en approche par compétences.

Des pratiques enseignantes documentées par Roblyer et Hughes (2019) qui s'inspirent du modèle RAT (Hughes, 2000, 2005) révèlent que l'intégration des technologies dans l'enseignement et dans l'évaluation donne lieu à plusieurs possibilités pour ce qui est de la réplication, de l'amplification et de la transformation des pratiques. Au-delà des possibilités qu'entraine l'usage des TN en enseignement, le cadre de l'évaluation des apprentissages à distance est peu connu et mérite d'être adapté afin de soutenir la compréhension des pratiques évaluatives en émergence au collégial. Étant donné que les évaluations des étudiants doivent rester rigoureuses en enseignement supérieur, les pratiques d'évaluation des apprentissages à distance méritent d'être analysées sous l'angle de l'intégration des technologies numériques. D'où notre question de recherche: Quelles sont les pratiques d'évaluation des apprentissages à distance mises en œuvre par des enseignants du collégial dans un programme en approche par compétences?

\section{L'évaluation des apprentissages à distance dans une approche par compétences}

Dans cette section, les dimensions du cadre de référence de l'évaluation des apprentissages à distance dans une approche par compétences et un modèle d'intégration des technologies numériques sont présentés.

\subsection{Un cadre de référence pour éclairer les pratiques évaluatives à distance dans une approche par compétences}

Afin de brosser un tableau des nombreuses dimensions qui sont interpellées pour évaluer des apprentissages à distance dans un programme en approche par compétences, nous présentons le cadre d'évaluation des apprentissages à distance qui a été conçu afin de comprendre les pratiques d'évaluation des apprentissages à distance d'enseignants en enseignement supérieur (Leroux, 2018). Ce cadre de référence a été construit sur la base d'une synthèse en deux niveaux. Le premier niveau comporte un approfondissement des résultats de recherches portant sur l'analyse des pratiques d'évaluation des compétences des enseignants du collégial (Leroux, 2010). Le deuxième niveau s'inspire de nos travaux antérieurs visant à dresser un bilan des pratiques évaluatives à distance en milieu universitaire (Nizet et al., 2016) et à proposer un processus d'évaluation des compétences en FAD (Leroux, Boyer, Corriveau, et Nolla, 2017).

Le cadre de référence présenté dans la figure 1 combine plusieurs dimensions qui sont présentées dans des rectangles qui s'emboitent les uns aux autres. Ces dimensions de l'évaluation des apprentissages à distance dans un programme en approche par compétences permettent d'apporter un éclairage sur les composantes et les paramètres à prendre en compte dans la structuration et la mise en œuvre des évaluations. 


\section{Figure 1}

Cadre de référence de l'évaluation des apprentissages à distance dans un programme en approche par compétences (Leroux, 2018)

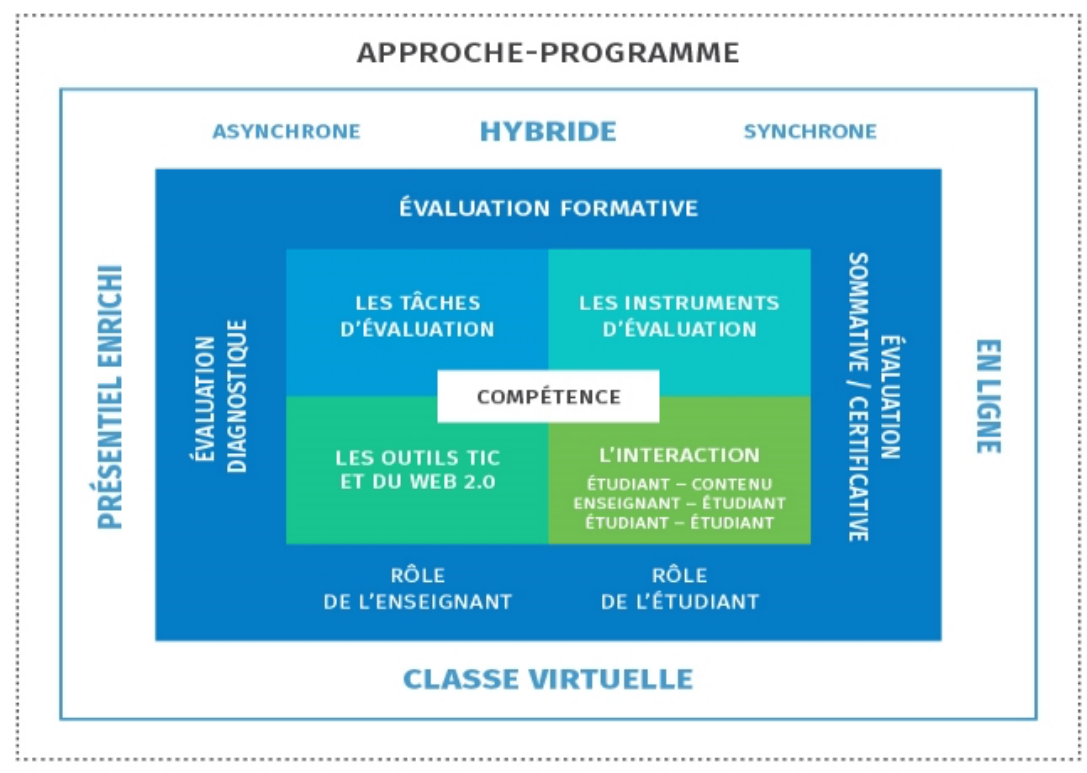

\subsubsection{L'APPROCHE-PROGRAMME}

Le premier rectangle, celui de l'approche-programme, présente la dimension la plus englobante. Cette dimension se caractérise par une ingénierie pédagogique (Basque, 2017) qui permet de prendre des décisions pédagogiques afin d'assurer une cohérence pédagogique à l'échelle du programme. L'approche-programme repose sur un projet de formation élaboré et poursuivi de manière collective par l'ensemble des acteurs impliqués dans le programme dans le but de développer une compréhension globale et commune des compétences développées dans chacun des cours du programme (Bélisle, 2015; Prégent, Bernard et Kozanitis, 2009).

\subsubsection{LE CONTEXTE D’ENSEIGNEMENT ET D'APPRENTISSAGE}

Le deuxième rectangle décline les contextes d'enseignement et d'apprentissage qui intègrent à différents degrés les TN : 1) en présentiel enrichi (face à face facilité par les TN), 2) hybride (activités en face à face et à distance) et 3) en ligne (toutes les activités se font à distance) (Allen et Seaman, 2013; Burton, Blais et Gilles, 2013; Meyer et Lakhal, 2017). Dans ces contextes, des activités d'enseignement et d'apprentissage peuvent se réaliser en mode synchrone (partager des informations en temps réel entre les participants) ou asynchrone (partage de communications en mode différé).

\subsubsection{L'INTENTION DE L'ÉVALUATION ET LES RÔLES}

Le troisième rectangle regroupe les intentions d'évaluation et les rôles de l'enseignant et de l'étudiant. Que ce soit dans une évaluation dont l'intention est de connaitre les acquis (évaluation diagnostique), de formuler des rétroactions ou de réguler les apprentissages (autoévaluation, coévaluation et évaluation mutuelle ou par les pairs) - évaluation formative - ou de rendre compte du degré d'acquisition de connaissances ou de compétences d'un étudiant - évaluation sommative/certificative -, l'enseignant et 
l'étudiant participent conjointement au processus d'évaluation en adoptant des rôles complémentaires (Allal, 2013; Leroux et Bélair, 2015; Mottier Lopez, 2015).

\subsubsection{LES DIMENSIONS DE L’ÉVALUATION DES APPRENTISSAGES À DISTANCE}

Le quatrième rectangle combine des dimensions à prendre en compte pour évaluer les compétences dans une situation d'apprentissage et d'évaluation qui intègre les TN. Les quatre dimensions comprennent :

1) les tâches d'évaluation caractérisées par leur complexité et leur authenticité (ex. : production écrite, communication orale, simulation, projets, etc.);

2) les instruments d'évaluation qui permettent de recueillir des traces afin de porter un jugement et de prendre une décision;

3) les outils TIC et du Web 2.0 qui permettent de médiatiser les activités d'enseignement et d'apprentissage;

4) l'interaction (enseignant-étudiant, étudiant-étudiant et étudiant-contenu) (Boettcher et Conrad, 2016; Josephsen, 2012; Jonsson, 2014; Ko et Rossen, 2017; Liang et Creasy, 2004; Lakhal et al., 2015; Nizet et al., 2016; Scallon, 2004; Stein et Graham, 2014; Stödberg, 2012; Tardif, 2006; Vai et Sosulski, 2015).

\subsection{Modèle d'intégration des technologies numériques}

L'intégration des TN à l'évaluation des apprentissages ouvre la porte à différents niveaux d'intégration des outils technologiques et de leur usage (Roblyer et Hughes, 2019). Les dimensions du modèle d'intégration des technologies permettent d'interpréter et de comprendre les évolutions possibles et la nature des transformations que les technologies entrainent dans les pratiques évaluatives des enseignants. De plus, étant donné que l'usage du numérique se répand aussi bien dans l'évaluation des compétences en présentiel qu'en FAD, le modèle de l'intégration du numérique permet de comprendre le sens (le pourquoi) ou le rationnel implicite des enseignants dans l'intégration des technologies dans les pratiques évaluatives. Le modèle d'intégration des technologies RAT développé par Hughes $(2000,2005)$ présente une taxonomie se déclinant en trois catégories distinctes. Ce modèle qui s'inspire du TPAK (Koehler, Mishra, Yahya et Yadav, 2004) a été retenu, puisqu'il s'appuie sur une recension des écrits au sujet de la transformation des pratiques enseignantes avec les TN et il a été validé par des données empiriques. 


\section{Figure 2}

Le modèle d'intégration des technologies RAT

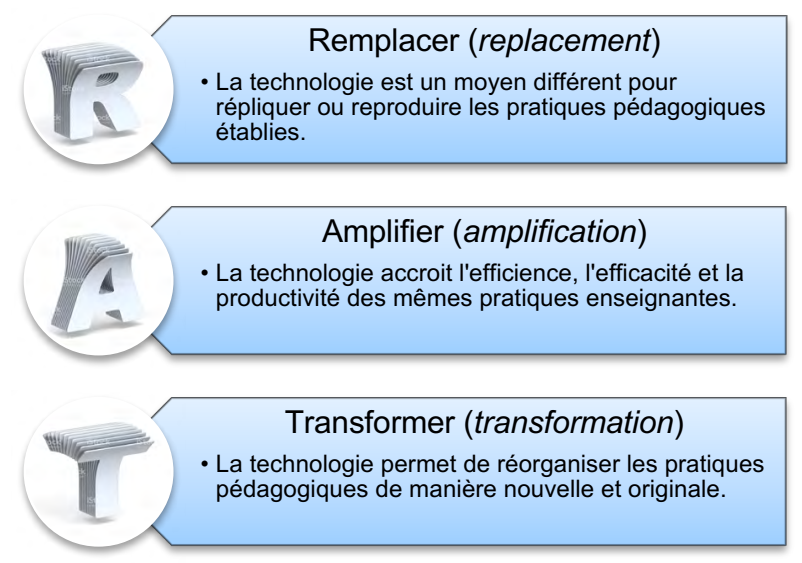

Note. Adapté de Hughes $(2000,2005)$ (traduction libre)

À travers ce modèle, l'auteur identifie des dimensions qui permettent d'examiner les aspects des activités d'apprentissage et d'évaluation dans laquelle l'utilisation des technologies est intégrée : 1) les méthodes d'enseignement qui comprennent l'évaluation des apprentissages, 2) les processus d'apprentissages des étudiants et 3 ) les objectifs du programme/contenu. Le tableau 1 présente les trois dimensions et les principaux thèmes qui contribuent à l'analyse des usages technologiques par les enseignants. Ce modèle permet de localiser les axes de transformations des pratiques enseignantes à travers l'intégration des technologies. Celui peut être utilisé pour comprendre les transformations entrainées par le numérique en évaluation, d'autant plus que les pratiques évaluatives constituent une composante essentielle de la pratique enseignante. 


\section{Tableau 1}

Dimensions pour guider l'analyse de l'utilisation des technologies (adapté de Hughes, 2000)

\begin{tabular}{|l|l|l|}
\hline Méthodes d'enseignement & \multicolumn{1}{|c|}{$\begin{array}{c}\text { Processus } \\
\text { d'apprentissage } \\
\text { de l'étudiant }\end{array}$} & $\begin{array}{c}\text { Objectifs du programme } \\
\text { /contenu }\end{array}$ \\
\hline $\begin{array}{l}\text { Le rôle de l'enseignant } \\
\text { Les activités ou les } \\
\text { tâches d'apprentissage }\end{array}$ & $\begin{array}{l}\text { Les connaissances à } \\
\text { acquérir, acquises ou à } \\
\text { appliquer }\end{array}$ \\
\hline $\begin{array}{l}\text { L'interaction avec les } \\
\text { étudiants } \\
\text { apprentissages }\end{array}$ & $\begin{array}{l}\text { Le processus de } \\
\text { réflexion - processus } \\
\text { cognitif }\end{array}$ & $\begin{array}{l}\text { Les expériences à acquérir, } \\
\text { acquises ou à exploiter }\end{array}$ \\
\hline $\begin{array}{l}\text { Le développement } \\
\text { professionnel }\end{array}$ & $\begin{array}{l}\text { Les modalités de } \\
\text { réalisation d'une tâche } \\
\text { (individuellement, petit } \\
\text { groupe, tout le groupe, } \\
\text { etc.) }\end{array}$ & $\begin{array}{l}\text { s. o. } \\
\text { l'étudiant }\end{array}$ \\
\hline $\begin{array}{l}\text { La planification de } \\
\text { l'enseignement }\end{array}$ & L'attitude de l'étudiant & s. o. \\
\hline $\begin{array}{l}\text { Les tâches administratives } \\
\text { relatives à l'enseignement }\end{array}$ & \multicolumn{1}{|c|}{ s. o. } & s. o. \\
\hline
\end{tabular}

Note. Ce tableau a été adapté des travaux de Hughes (2000; traduction libre).

La catégorie Remplacer (replacement) implique un usage des technologies pour substituer et, en aucun cas, modifier les pratiques pédagogiques établies, les processus d'apprentissage des étudiants ou les objectifs de contenu. La technologie est utilisée dans une logique ou avec une intention de reproduction ou de transposition des méthodes d'évaluation usuelles. En ce sens, la technologie est intégrée comme un moyen différent afin de reproduire ou de répéter les mêmes pratiques en lien avec les objectifs d'apprentissage du programme. Dans cette catégorie, aucune modification des pratiques évaluatives établies et de la participation de l'étudiant n'est observée. Du point de vue de l'enseignant et des étudiants, la valeur ajoutée de la technologie est nulle dans ce type d'utilisation, puisque le recours à la technologie n'apporte en effet aucun changement dans les pratiques d'enseignement et la participation de l'étudiant. De plus, le rôle de l'enseignant reste le même tout au long du processus d'enseignement et d'apprentissage. En ce sens, la motivation de l'étudiant ne connait aucune amélioration. De plus, on n'observe aucun gain en termes d'économie de temps. À titre d'exemple, un examen est réalisé en ligne sur la plateforme Moodle avec les mêmes types de questions que l'examen administré en salle de classe (papier et crayon).

Dans la catégorie Amplifier, l'accent est mis sur l'efficacité ou sur la rationalisation de l'utilisation de la technologie qui amplifie les pratiques pédagogiques et évaluatives existantes. En ce sens, l'amplification correspond à un ajustement stratégique en vue de réaliser les mêmes objectifs tout en réduisant les coûts en termes d'efforts consentis. À titre d'exemple, des rétroactions audio et vidéo plus fréquentes sont 
formulées rapidement. Ces rétroactions offrent plus de détails Les TN sont utilisés pour améliorer les processus existants. Leur utilisation va permettre à l'enseignant d'agir d'une manière optimale, soit pour gagner un peu de temps, soit pour améliorer le niveau d'engagement des étudiants, sans que des changements substantiels ne soient observés dans les orientations de son enseignement, des contenus et des objectifs du programme. À titre d'exemple, des rétroactions audio et vidéo plus fréquentes sont formulées rapidement. Ces rétroactions offrent plus de détails.

La logique de la catégorie Transformer requiert une réorganisation des pratiques évaluatives. Cette réorganisation implique que des changements importants soient pris en compte afin de proposer des pratiques nouvelles. En somme, la logique de la transformation exige que l'intégration des TN ait un impact sur les méthodes pédagogiques, le processus d'apprentissage de l'étudiant, les contenus d'apprentissage et parfois sur les objectifs de la formation. À titre d'exemple, la vidéo est utilisée lors d'une simulation afin que l'étudiant puisse se filmer et annoter sa performance. Sa production annotée sera utilisée afin de réaliser une autoévaluation à l'aide d'une grille d'évaluation critériée.

\section{Objectif spécifique et méthodologie de recherche}

En prenant appui sur les pratiques évaluatives d'enseignants du collégial, l'objectif spécifique de la recherche est d'analyser les pratiques d'évaluation formatives et sommatives/certificatives à distance d'enseignants dans le cadre d'un cours. Considérant la nature de l'objectif de recherche, une méthodologie de type qualitatif/interprétatif (Savoie-Zajc, 2011) semble la plus appropriée pour comprendre les pratiques évaluatives des apprentissages d'enseignants qui évaluent les apprentissages à distance au collégial.

Les participants à cette recherche sont des enseignants du collégial reconnus par des pairs pour avoir intégré des méthodes d'évaluation des apprentissages à distance dans le cadre d'un cours à la formation régulière ou continue, dans un programme préuniversitaire ou technique. II s'agit d'un échantillonnage non probabiliste, par choix raisonné (Fortin et Gagnon, 2015) dont la sélection est basée sur la réputation d'après la recommandation d'un informateur clé, soit des conseillers pédagogiques des collèges (Miles et Huberman, 2003). Un cadre d'échantillonnage explicite (Leroux, 2010) nous a permis de sélectionner 14 enseignants du collégial provenant de 6 établissements partageant un ensemble de caractéristiques similaires. Ainsi, nous prendrons un échantillon total de 14 enseignants. Cet échantillon nous apparait suffisant compte tenu des ressources disponibles dans le cadre du concours. Le terrain de recherche comprendra l'ensemble des collèges francophones du réseau collégial. Le choix de ce terrain de recherche relève du fait que les programmes des collèges sont basés sur l'APC. De plus, le fait que la chercheuse principale participe au secteur Performa, qui regroupe 61 collèges partenaires, facilitera l'accès aux enseignants des collèges.

Les données recueillies proviennent d'entrevues semi-dirigées (Savoie-Zajc, 2004) et de la collecte de documents en lien avec le cours (plan de cours et documents d'évaluation formels présentés en fichier électronique). Un guide d'entrevue semi-dirigée composé de questions ouvertes a été élaboré pour permettre aux enseignants d'expliquer de manière détaillée leurs pratiques d'évaluation des apprentissages à distance. La formulation des questions a été guidée par le cadre de référence de l'évaluation des apprentissages à distance dans une approche par compétences. Le guide d'entrevue semi-dirigée a été adapté de Leroux (2010) et de Nizet et al. (2016). Ce guide prévoit un certain nombre de questions principales qui servent de points de repère et des sous-questions afin de relancer le participant au besoin. 
La collecte de données s'est échelonnée du mois d'avril 2017 au mois de janvier 2018. Les entrevues semi-dirigées, d'une durée de 90 à 120 minutes, ont été enregistrées à l'aide de l'outil de télécollaboration Cisco WebEx. Les documents en lien avec le déroulement logique du cours ont été recueillis et sauvegardés dans un dossier pour chacun des participants.

Dans le but de saisir le sens des données recueillies, une démarche d'analyse inductive modérée (SavoieZajc, 2004) a été effectuée à partir des dimensions du cadre de référence de l'évaluation des apprentissages à distance dans un programme en approche par compétences. À partir des dimensions du cadre de référence, l'analyse des données qualitatives a été effectuée. D'abord, le codage des données a été réalisé, en suivant les étapes proposées par Van der Maren (1996). Le contenu des données audio et vidéo a d'abord été retranscrit in extenso en données de recherche. Une condensation des données a ensuite été effectuée par une démarche de codage, de repérage et de catégorisation des passages significatifs, sélectionnée selon les étapes de l'analyse et du traitement, à l'aide du logiciel NVivo 11 (Benght et McDougall, 2017). La validité du travail d'analyse et d'interprétation a été fondée sur les démarches d'intracodage et d'intercodage (Miles et Huberman, 2003).

\section{Des pratiques d'évaluation formative et sommative/certificative}

De manière fine, en sondant en profondeur les pratiques d'évaluation des apprentissages pour un cours, la présentation des résultats met en perspective les pratiques rapportées de deux enseignants qui partagent des expériences et des éléments communs : première expérimentation du cours choisi à distance, clientèle hétérogène, conception des activités d'apprentissage et d'évaluation par l'enseignant, programme de formation technique. Les pratiques évaluatives seront succinctement décrites puis détaillées dans un tableau-synthèse regroupant les principaux éléments qui se dégagent des dimensions de l'évaluation des apprentissages à distance pour le cours ciblé.

\subsection{Des pratiques évaluatives qui engagent l'étudiant dans la réalisation de tâches authentiques}

Dans le cadre du dernier cours d'un programme technique visant le développement de trois compétences, Odile, qui a offert un cours en présence à plusieurs reprises, offre pour la première fois un cours à distance. Dans un contexte d'enseignement et d'apprentissage totalement en ligne et en mode synchrone, le cours ciblé par Odile propose un projet synthèse « qui vient un peu reprendre ce qui a été vu dans les autres cours [...]. Donc, c'est un cours intégrateur; d'une durée de 60 heures » (O. 19-22). Malgré son expérience dans l'enseignement de ce cours en salle de classe, Odile rapporte ses pratiques évaluatives dans le cours qu'elle donne pour la première fois totalement en ligne, en mode synchrone. Les activités d'apprentissage et d'évaluation, en combinant la plateforme numérique Blackboard Collaborate qui permet d'offrir une classe virtuelle et la plateforme Moodle, offrent un accès à l'ensemble de la documentation des activités d'apprentissage et d'évaluation du cours.

Malgré l'inconfort lié à la mise en ligne du cours et les nombreux défis liés à l'accompagnement d'un projet en ligne, afin de soutenir le développement des compétences dans le cours qui se situe au terme du programme, Odile a misé sur l'identification d'un projet réel ou fictif par étudiant. L'enseignante rapporte : 
« j'ai choisi de proposer un projet malgré un peu d'inconfort au début, mais j'avais envie de le faire. Je laissais aux étudiants le libre choix du projet [...]. Ils avaient beaucoup de liberté sur le format de remise ou sur le contenu qu'ils allaient produire » (0. 90-96). Également, en lien avec la thématique du projet, elle demandait aux étudiants de produire un article à déposer sur un blogue de la classe.

De manière à soutenir l'évaluation formative en cours de réalisation des deux tâches complexes et authentiques, l'enseignante a prévu plusieurs modalités de rétroactions et de régulations. Également, un quiz est proposé à la mi-session afin de « faire le point [...] sur ce qu'on sait et ce qui a été oublié. » (O. 10561063). Comme en témoigne l'enseignante, une coévaluation a été prévue à chacune des étapes du projet " qui se divisait en sept jalons » (O. 122). Ces coévaluations permettaient d'apprécier de manière réaliste la durée de réalisation du projet et de chacune des étapes, et d'ajuster l'ampleur du projet. Sur une base hebdomadaire, chaque étudiant remplissait une feuille de temps dans un fichier modèle partagé sur Google Drive qui permettait l'autoévaluation et l'autorégulation des réalisations des étapes du projet par l'étudiant. Au sujet de l'évaluation de l'article, Odile précise que l'évaluation mutuelle proposée a contribué à la formulation de nombreuses rétroactions : « Chaque étudiant avait l'article de deux personnes à commenter et ils recevaient les commentaires de deux personnes. J'étais un peu étonnée de voir qu'ils se répondaient. Je pensais que cela tomberait un peu à plat et [...] qu'ils feraient cette activité pour me faire plaisir. Mais, finalement, il y a eu plusieurs d'échanges et ils se répondaient. Et, à la suite de ça, ils faisaient leur maquette et la présentaient aux autres étudiants. Alors, qu'il n'y avait pas de points associés [...] à la présentation » (O. 352-356).

Afin d'évaluer les compétences du cours, l'évaluation sommative/certificative des compétences prévoit deux tâches qui se déroulent en plusieurs étapes. Des grilles d'évaluation à échelle uniforme ont été conçues par l'enseignante afin de porter un jugement sur chacune des étapes du projet et sur l'article.

À partir de ce qu'Odile a dit et des observations en lien avec le cours choisi, dans le tableau 2, les dimensions de la pratique évaluative des apprentissages à distance totalement en ligne sont présentées. 


\section{Tableau 2}

Les dimensions de la pratique d'évaluation des apprentissages à distance totalement en ligne d'Odile

\begin{tabular}{|c|c|}
\hline Dimensions & Description \\
\hline Approche-programme & $\begin{array}{l}\text { - Programme technique, formation continue, cours de } 60 \text { heures, cours } \\
\text { intégrateur au terme du programme }\end{array}$ \\
\hline $\begin{array}{l}\text { Contexte d'enseignement } \\
\text { et d'apprentissage }\end{array}$ & $\begin{array}{l}\text { - En ligne : cours totalement à distance } \\
\text { - Modalité synchrone et asynchrone }\end{array}$ \\
\hline Intention d'évaluation & $\begin{array}{l}\text { Évaluation formative : } \\
\text { - Rétroactions : verbales, écrites, tableaux ou graphiques et audio } \\
\text { - Évaluation mutuelle d'un article } \\
\text { - } \text { Autoévaluation de sa production avec la grille d'évaluation } \\
\text { - Autorégulation : ajuster les étapes de réalisation de son projet } \\
\text { Euiz } \\
\text { Évaluation sommative/certificative } \\
\text { - Projet (étapes) : planification, stratégie de communication, design } \\
\text { (s'inspirer, commenter, produire et présenter, ajuster), réalisation et } \\
\text { - } \text { mise en ligne de la documentation, présentation finale } \\
\text { - Rédaction d'un article (blogue) }\end{array}$ \\
\hline Rôle de l'enseignant & - Enseignant : accompagnateur, guide collaborateur \\
\hline Rôle de l'étudiant & - Étudiant : autonomie, contre vérificateur, professionnalisme \\
\hline Tâches d'évaluation & $\begin{array}{l}\text { - Réaliser et présenter un projet de maquette } \\
\text { - Rédiger un article (blogue) }\end{array}$ \\
\hline Instruments d'évaluation & $\begin{array}{l}\text { - Grille d'évaluation à échelle uniforme } \\
\text { - Quiz (réponses courtes) }\end{array}$ \\
\hline Outils TIC et du Web 2.0 & $\begin{array}{l}\text { - } \text { Blackboard : classe virtuelle } \\
\text { - } \text { Moodle : plateforme numérique d'apprentissage (plan de cours, } \\
\text { - } \text { Google Drive : suivis du projet } \\
\text { - } \quad \text { Acrice : Word, PPT } \\
\text { - } \quad \text { Intranet : plateforme institutionnelle (remise des notes et messagerie) } \\
\text { - Facebook : communication entre les étudiants }\end{array}$ \\
\hline Interaction & $\begin{array}{l}\text { - Étudiant-contenu : projet } \\
\text { - Enseignant-étudiant : individuel et groupe } \\
\text { - Étudiant-étudiant : évaluation mutuelle (dyade) }\end{array}$ \\
\hline
\end{tabular}




\subsection{Des pratiques évaluatives qui misent sur l'efficacité dans un contexte hybride}

Dans le cadre d'un cours d'un programme technique qui accueille une clientèle hétérogène, Nicolas dévoile les pratiques évaluatives afin d'évaluer la compétence du cours. Le cours de 45 heures est donné sur plus d'un site (multisites) prévoit 15 heures de cours en mode synchrone, 15 heures de cours en mode asynchrone et 15 heures de cours en présence afin de réaliser les laboratoires.

Nicolas donne ce cours pour la première fois. D'entrée de jeu, il souligne l'importance des technologies numériques afin de soutenir l'évaluation des apprentissages des nombreux cours et " d'accélérer le processus d'évaluation et d'enseignement [...]. Je pense que sans ces technologies, à la bonne vieille méthode, je n'aurais peut-être pas été capable d'en faire autant » (N. 21-26). Parallèlement à l'intégration des technologies numériques, cet enseignant s'interroge sur l'ajustement de ses pratiques évaluatives à l'approche par compétences. Cet enseignant soutient qu'il a réussi à diminuer le morcellement de l'évaluation de la compétence. Comme l'explique Nicolas, l'analyse de la compétence du cours a permis d'identifier un processus en huit étapes qui se répète pour chacune des espèces ciblées par le cours. Ainsi, " j'en suis venu à évaluer la compétence au complet à chaque évaluation, mais pour une espèce différente » (N. 294-297). Afin d'évaluer la compétence du cours, l'enseignant ne se limite pas à l'examen. II propose la réalisation d'un projet et d'un compte rendu, d'une communication orale et des rapports et procédures de laboratoire.

Afin de soutenir l'évaluation formative des apprentissages, Nicolas souligne l'apport les technologies qui ont permis de formuler de nombreuses rétroactions écrites automatiques : "J'utilise souvent les Google Forms qui donnent directement aux étudiants la réponse ou la correction à faire. [...] Ça permet de faire beaucoup plus de formatifs avec une correction [...] automatique » (N. 324-329). Également, il souligne que les étudiants ont reçu de nombreuses rétroactions verbales tout au long du cours à travers les moments de réalisation des tâches complexes. Ses pratiques évaluatives témoignent de la participation active des étudiants lors de l'autoévaluation du modèle (projet), la coévaluation du rapport de laboratoire avec l'enseignant et l'évaluation mutuelle de la communication orale présentée par un pair.

Pour évaluer les tâches complexes, Nicolas a conçu une grille d'évaluation à échelle globale. Pour diminuer la tricherie en contexte d'évaluation sommative/certificative, l'enseignant administre les questions de connaissances qui sont posées lors d'une rencontre en mode synchrone avec chaque étudiant sur Vidyo. Également, Nicolas implique les étudiants dans la formulation de questions. Avec les technologies numériques dont il dispose pour soutenir l'enseignement et l'évaluation des apprentissages, Nicolas combine les TN à travers le processus d'évaluation des apprentissages à distance en favorisant la médiatisation des contenus et des interactions entre les acteurs impliqués.

À partir de ce que Nicolas a dit et d'observations en lien avec le cours choisi, dans le tableau 3, les dimensions de la pratique évaluative des apprentissages à distance totalement en mode hybride sont présentées. 


\section{Tableau 3}

Les dimensions de la pratique d'évaluation des apprentissages à distance de Nicolas dans un contexte hybride (multisites)

\begin{tabular}{|c|c|}
\hline Dimensions & Description \\
\hline Approche-programme & $\begin{array}{l}\text { - Programme technique, formation spécifique, cours de } 45 \text { heures, cours } \\
\text { obligatoire }\end{array}$ \\
\hline $\begin{array}{l}\text { Contexte d'enseignement } \\
\text { et d'apprentissage }\end{array}$ & $\begin{array}{l}\text { - Hybride : cours en télé-enseignement ( } 15 \text { heures), } 15 \mathrm{~h} \text { asynchrone et } \\
15 \mathrm{~h} \text { laboratoire. } \\
\text { - Modalités synchrone et asynchrone }\end{array}$ \\
\hline Intention d'évaluation & $\begin{array}{l}\text { Évaluation formative: } \\
\text { - Rétroactions verbales, écrites et automatisées } \\
\text { - } \text { Cotoévaluation de son modèle (projet) } \\
\text { - Évaluation mutuelle de la communication orale présentée par un pair } \\
\text { Évaluation sommative/certificative } \\
\text { - Projet et compte rendu } \\
\text { - Communication orale } \\
\text { - Laboratoires } \\
\text { - Examen oral }\end{array}$ \\
\hline Rôle de l'enseignant & - Enseignante : accompagnateur, guide, collaborateur \\
\hline Rôle de l'étudiant & - Étudiant : actif, collaborateur, évaluateur \\
\hline Tâches d'évaluation & $\begin{array}{l}\text { - Projet et compte rendu } \\
\text { - } \quad \text { Présentation d'articles scientifiques : communication orale } \\
\text { - }\end{array}$ \\
\hline Instruments d'évaluation & $\begin{array}{l}\text { - Examens (mises en situation, questions à développement, calculs, } \\
\text { procédures) } \\
\text { - Grille d'évaluation à échelle globale }\end{array}$ \\
\hline Outils TIC et du Web 2.0 & $\begin{array}{l}\text { - Vidyo : classe virtuelle } \\
\text { - } \quad \text { GouTube : vidéo } \\
\text { - } \quad \text { Soogle suite (Google Doc, Google Forms, Google Sheet, Google Slide, } \\
\text { - Screencast-O-Matic : enregistrement vidéo des présentations } \\
\text { - Intranet : plateforme institutionnelle (remise des notes et messagerie) }\end{array}$ \\
\hline Interaction & $\begin{array}{l}\text { - Étudiant-contenu : projet, laboratoire, articles } \\
\text { - Enseignant-étudiant : individuel } \\
\text { - Enseignant-étudiant : groupe } \\
\text { - Étudiant-étudiant : évaluation mutuelle }\end{array}$ \\
\hline
\end{tabular}




\section{De la réplication à la transformation des pratiques d'évaluation}

Le modèle de Hugues $(2000,2005)$ permet de révéler les pratiques d'évaluation à distance selon la catégorisation des différents niveaux de changements pour les trois dimensions. L'intégration des TN en enseignement comporte des moments de réplication, d'amplification et de transformation, sans qu'il y ait un ordre linéaire dans le passage d'une catégorie à une autre (Roblyer et Hughes, 2019).

Les pratiques rapportées par Odile exposent l'intégration des TN dans un cours totalement en ligne. Les résultats présentés dans le tableau 4 démontrent que cette enseignante est arrivée non seulement à améliorer l'efficacité de ses pratiques en intégrant les TN dans le cours en ligne, mais aussi à redéfinir certaines d'entre elles pour les transformer. L'intégration des TN a permis de répliquer des pratiques d'évaluation formative des connaissances et d'assurer la rigueur du processus d'évaluation sommatif/certificatif en utilisant les espaces sécurisés et soutenues par l'établissement. L'amplification des pratiques est repérable par l'augmentation de la fréquence des rétroactions individualisées formelles et informelles lors de la réalisation du projet et l'utilisation d'un blogue afin de rédiger et de partager un article. L'intégration des TN a également contribué à la transformation de pratiques, puisque ces TN ont permis de trouver de nouvelles solutions afin de proposer une évaluation sommative/certificative qui comprend deux tâches authentiques. Ils ont contribué à redéfinir les modalités de rétroactions et de régulations qui engagent les étudiants. En plus de solliciter l'autonomie de l'étudiant dans ses apprentissages, les modalités de réalisation du projet ont engagé l'étudiant dans la recherche et la consultation de ressources en ligne afin de trouver des solutions aux problèmes rencontrés. 


\section{Tableau 4}

L'intégration des TN par Odile dans un cours incluant des activités d'évaluation totalement à distance

\begin{tabular}{|c|c|c|c|}
\hline & Méthodes d'évaluation & Apprentissages & Programme \\
\hline $\begin{array}{l}\text { Remplacement } \\
\text { La technologie est un } \\
\text { moyen différent } \\
\text { d'atteindre le même } \\
\text { objectif. }\end{array}$ & $\begin{array}{l}\text { Un quiz formatif est } \\
\text { utilisé à la mi-session } \\
\text { pour évaluer les } \\
\text { connaissances. } \\
\text { Les informations } \\
\text { relatives au cours sont } \\
\text { communiquées dans les } \\
\text { espaces sécurisés : } \\
\text { Moodle et intranet de } \\
\text { l'établissement. }\end{array}$ & s. 0. & s. 0. \\
\hline $\begin{array}{l}\text { Amplification } \\
\text { La technologie } \\
\text { augmente ou intensifie } \\
\text { l'efficacité, la } \\
\text { productivité, l'accès, les } \\
\text { capacités, etc., mais les } \\
\text { tâches restent } \\
\text { fondamentalement les } \\
\text { mêmes. }\end{array}$ & $\begin{array}{l}\text { Le projet et l'article sont } \\
\text { réalisés } \\
\text { individuellement. } \\
\text { Le blogue est utilisé } \\
\text { pour rédiger un article } \\
\text { sur trois sites inspirants. } \\
\text { Les rétroactions sur le } \\
\text { projet sont redéfinies à } \\
\text { l'aide d'une feuille de } \\
\text { temps partagée avec } \\
\text { l'étudiant sur Google } \\
\text { Drive. }\end{array}$ & s. o. & $\begin{array}{l}\text { L'évaluation des } \\
\text { apprentissages cible les } \\
\text { trois compétences du } \\
\text { cours intégrateur. } \\
\text { Les connaissances } \\
\text { essentielles sont } \\
\text { évaluées dans une } \\
\text { évaluation formative }\end{array}$ \\
\hline $\begin{array}{l}\text { Transformation } \\
\text { La technologie redéfinit, } \\
\text { restructure, réorganise, } \\
\text { change ou crée de } \\
\text { nouvelles solutions. }\end{array}$ & $\begin{array}{l}\text { Un projet de maquette } \\
\text { est réalisé totalement en } \\
\text { ligne par chaque } \\
\text { étudiant. } \\
\text { L'étudiant participe à } \\
\text { l'autoévaluation, la } \\
\text { coévaluation de son } \\
\text { projet et à l'évaluation } \\
\text { mutuelle de l'article } \\
\text { déposé sur le blogue. }\end{array}$ & $\begin{array}{l}\text { Les modalités de } \\
\text { réalisation du projet de } \\
\text { maquette avec les outils } \\
\text { et ressources } \\
\text { technologiques } \\
\text { engagent } \\
\text { individuellement chaque } \\
\text { étudiant qui effectue } \\
\text { librement le choix de } \\
\text { son projet. } \\
\text { L'étudiant doit } \\
\text { démontrer de } \\
\text { l'autonomie pour mener } \\
\text { son projet et trouver des } \\
\text { solutions aux problèmes } \\
\text { rencontrées en } \\
\text { consultant des } \\
\text { ressources en ligne. }\end{array}$ & s. 0 . \\
\hline
\end{tabular}

Les pratiques rapportées par Nicolas mettent en exergue l'intégration des TN dans un cours offert dans un contexte d'enseignement et d'apprentissage hybride sur plusieurs sites (multisites). Dans les résultats présentés dans le tableau 5, l'enseignant rapporte que le travail d'analyse de la compétence du cours avec le conseiller pédagogique a contribué à cibler l'évaluation d'un processus qui est repris plusieurs fois dans 
le cadre du cours qui aborde " 5 ou 6 espèces différentes » (N. 100). Cette prise en compte d'une évaluation du processus de la compétence combinée avec l'intégration des TN dans un contexte hybride (multisites) ont amené l'enseignant à proposer des pratiques d'évaluation formative et sommative/certificative de connaissances et de les combiner avec des évaluations plus complexes qui nécessitent l'utilisation de plusieurs outils technologiques.

Comme le souligne l'enseignant, l'intégration des TN lui a permis d'augmenter l'efficacité de l'évaluation des apprentissages : « Je pouvais faire des corrections automatisées des évaluations formatives qui me permettaient de ne pas avoir à corriger chaque copie individuellement. Ensuite, je pouvais consulter les statistiques pour cibler les questions qui ont été moins réussies et y revenir au prochain cours. Donc, c'était une accélération [de ma pratique], je pouvais synthétiser rapidement » (N. 982-990). L'intégration des TN a permis de proposer des tâches complexes (projet et compte rendu, communication orale, rapports et procédures en laboratoire), de proposer un examen oral individualisé en mode synchrone (Vidyo) et de proposer de nombreuses rétroactions écrites et automatisées. Cette amplification des pratiques a permis d'augmenter la rapidité et la fréquence des rétroactions individualisées formelles et informelles, des examens et des tâches complexes. La combinaison de l'ensemble des TN a contribué à la transformation de pratiques pour évaluer les compétences du cours, puisqu'elle a permis de trouver de nouvelles solutions afin de proposer des évaluations formatives et sommative/certificative dans un cours qui est offert sur plusieurs sites (multisites). En plus de solliciter l'autonomie de l'étudiant dans ses apprentissages, les modalités de réalisation du projet et les modalités de rétroactions et de régulations (autoévaluation, coévaluation et évaluation mutuelle) ont engagé l'étudiant dans l'évaluation de ses apprentissages. 


\section{Tableau 5}

L'intégration des TN par Nicolas dans un cours hybride (multisites)

\begin{tabular}{|c|c|c|c|}
\hline & Méthodes d'évaluation & Apprentissages & Programme \\
\hline $\begin{array}{l}\text { Remplacement } \\
\text { La technologie est un } \\
\text { moyen différent } \\
\text { d'atteindre le même } \\
\text { objectif. }\end{array}$ & $\begin{array}{l}\text { Des examens sont } \\
\text { proposés (Google Forms). } \\
\text { Les informations relatives } \\
\text { au cours et aux résultats } \\
\text { sont communiquées dans } \\
\text { les espaces sécurisés: } \\
\text { intranet de } \\
\text { l'établissement. }\end{array}$ & s. o. & s. o. \\
\hline $\begin{array}{l}\text { Amplification } \\
\text { La technologie } \\
\text { augmente ou intensifie } \\
\text { l'efficacité, la } \\
\text { productivité, l'accès, les } \\
\text { capacités, etc., mais les } \\
\text { tâches restent } \\
\text { fondamentalement les } \\
\text { mêmes. }\end{array}$ & $\begin{array}{l}\text { Les tâches complexes } \\
\text { ciblées sont réalisées } \\
\text { individuellement ou en } \\
\text { équipe (Google). } \\
\text { Un examen oral et } \\
\text { individualisé : questions } \\
\text { de connaissances } \\
\text { Rétroaction écrite (Google } \\
\text { Sheet) } \\
\text { La correction automatisée } \\
\text { des examens avec Google } \\
\text { Forms permet de donner } \\
\text { des évaluations formatives } \\
\text { plus fréquentes. }\end{array}$ & s. o. & $\begin{array}{l}\text { L'évaluation des } \\
\text { apprentissages cible le } \\
\text { processus (étapes) de } \\
\text { la compétence. } \\
\text { Les connaissances sont } \\
\text { évaluées dans une } \\
\text { intention formative et } \\
\text { parfois sommative. }\end{array}$ \\
\hline $\begin{array}{l}\text { Transformation } \\
\text { La technologie redéfinit, } \\
\text { restructure, réorganise, } \\
\text { change ou crée de } \\
\text { nouvelles solutions. }\end{array}$ & $\begin{array}{l}\text { La technologie permet de } \\
\text { réorganiser les activités } \\
\text { d'apprentissage et } \\
\text { d'évaluation du cours qui } \\
\text { est offert sur plusieurs } \\
\text { sites (multisites). } \\
\text { L'étudiant } \\
\text { participe à l'autoévaluation } \\
\text { de son projet, la } \\
\text { coévaluation du rapport de } \\
\text { laboratoire et l'évaluation } \\
\text { mutuelle de la } \\
\text { communication orale } \\
\text { présentée par un pair. }\end{array}$ & s. o. & s. o. \\
\hline
\end{tabular}




\section{Des pratiques d'évaluation des apprentissages à distance en transformation}

L'intégration des technologies numériques (TN) à l'évaluation des apprentissages dans un programme en approche par compétences en enseignement supérieur introduit de nouvelles tendances (Louis et Bédard, 2015). Les résultats de la recherche permettent de soulever deux constats.

\subsection{Des pratiques évaluatives diversifiées qui combinent plusieurs méthodes}

Les résultats de la recherche mettent en relief un premier constat: les pratiques d'évaluation des apprentissages à distance sont innovantes (Lison, Bédard, Beaucher, et Trudelle, 2014) et diversifiées. D'un enseignant à un autre, les pratiques d'évaluation sont en effet loin d'être les mêmes. Cette diversité des pratiques s'explique d'abord par la planification et la mise en œuvre de pratiques d'évaluation des apprentissages à distance qui font appel à des ressources pédagogiques, humaines, technologiques et matérielles qui sont variables d'un établissement à l'autre (Leroux et al., 2019). Également, l'autonomie des établissements en matière d'évaluation des apprentissages et sur le plan de la mise en œuvre des programmes en approche par compétences introduit de nombreux paramètres que les enseignants doivent prendre en compte (Leroux, 2010). Malgré cette diversité de pratiques évaluatives, les résultats révèlent que les pratiques évaluatives semblent tenir compte des politiques institutionnelles d'évaluation des apprentissages (PIEA) d'établissement et les décisions prises à l'échelle du programme (Bélisle, 2015; Leroux et al., 2019).

Les pratiques rapportées relèvent une diversité de pratiques d'évaluation formative qui comprend la correction automatisée, les modalités de rétroactions et de régulation (autoévaluation, coévaluation et évaluation mutuelle ou par les pairs). En plus de favoriser l'interaction à travers le processus d'évaluation des apprentissages, ces pratiques d'évaluation formatives témoignent de pratiques émergentes qui contribuent à fournir de façon rapide et efficace une rétroaction ciblée, pertinente et utile à l'étudiant au cours du processus d'exécution de la tâche et à l'enseignant par la suite (Leroux, 2018; Louis et Bédard, 2015). Les pratiques des enseignants confirment la participation de l'étudiant à l'évaluation de ses apprentissages afin de soutenir le développement de la compétence.

Dans les cours ciblés, les pratiques d'évaluation rapportées par les enseignants ne se limitent pas à la correction automatisée de tests et d'examens. Les pratiques d'évaluation sont diversifiées et font appel à une combinaison de méthodes d'évaluation tout en favorisant l'interaction (étudiant-contenu, étudiantenseignant et étudiant-étudiant). Alors que l'évaluation sommative/certificative fait appel principalement à la démonstration de compétence, les tâches complexes et authentiques sont proposées et elles sont réalisées principalement individuellement en mobilisant de nombreuses ressources et une diversité d'outils technologiques (Kozanitis, 2021 ; Lakhal et al.,2015; Nizet et al., 2016).

\subsection{Une intégration variable des technologies à l'évaluation des apprentissages}

Les résultats montrent que les pratiques rapportées sollicitent une intégration et une combinaison variable et complexe des technologies numériques (TN) pour évaluer les compétences et les connaissances ciblées par le cours. Bien que les outils technologies soient différents, les enseignants accompagnent les étudiants afin de s'assurer de l'accessibilité et de la maitrise des outils lors des activités d'évaluation des apprentissages. 
Le modèle RAT (Hughes, 2000, 2005) a permis de révéler les catégories d'intégration des TN en fonction de trois dimensions : les méthodes pédagogiques, le processus d'apprentissage de l'étudiant, ainsi que les objectifs du programme et les contenus de formation. Dans la catégorie de remplacement (R), les enseignants ont recours aux TN dans une logique de reproduction des pratiques d'évaluation usuelles. Les résultats de la recherche confirment que les enseignants privilégient des pratiques de remplacement lors de l'administration de méthodes d'évaluation dites plus traditionnelles (quiz, examen). Également, ils utilisent les TN afin de communiquer les informations au sujet de l'évaluation des apprentissages (ex. : rétroactions formelles, grilles d'évaluation, décisions, résultats) dans les espaces sécurisés et supportés par l'établissement (ex. : Moodle, intranet).

Dans la catégorie d'amplification $(A)$, les résultats démontrent que l'efficacité des pratiques d'évaluation est amplifiée par l'intégration des TN. Lors de la proposition de tâches complexes, l'usage d'outils technologiques favorise la collaboration et le partage continu et rapide de l'information. En plus d'améliorer le processus existant et de gagner du temps, ces outils favorisent l'interaction et la participation active de l'étudiant à travers le processus d'évaluation. Sans qu'il y ait de changements substantiels sur le plan des objectifs du programme/contenu, les résultats confirment que l'intégration des TN agit comme une loupe grossissante qui amène l'enseignant à préciser sa compréhension de la compétence à développer, des connaissances essentielles à acquérir et des méthodes d'évaluation à privilégier (Leroux et al., 2019).

La troisième catégorie s'inscrit dans une logique de transformation $(T)$ : les TN permettent de réorganiser de manière nouvelle l'évaluation des apprentissages. Les résultats de recherche mettent en exergue une réorganisation du déroulement d'activités d'apprentissage et d'évaluation dans le cadre d'un cours (hybride et en ligne). Les résultats confirment que les TN ont permis aux deux enseignants de proposer de nouvelles occasions de réaliser l'entièreté de tâches complexes et authentiques à travers un processus d'accompagnement par l'enseignant favorisant l'interaction, la rétroaction et la régulation.

\section{Conclusion}

La présente recherche vise à identifier des pratiques d'évaluation formative et sommative/certificative des apprentissages à distance d'enseignants du collégial dans un programme en approche par compétences.

Les pratiques rapportées par les deux enseignants confirment qu'ils intègrent les TN dans des activités pédagogiques offertes en présence, totalement à distance ou selon un mode hybride qui privilégie des modalités de communication synchrone et asynchrone. Dans ces contextes, les pratiques d'évaluation ne se limitent pas à la correction automatisée de tests et d'examens. Les pratiques d'évaluation sont diversifiées et font appel à une combinaison de méthodes d'évaluation (examen, production écrite, démonstration de compétences), d'instruments (grille d'évaluation), d'outils technologiques et de nombreuses interactions (étudiant-contenu, étudiant-enseignant et étudiant-étudiant). Alors que l'évaluation sommative/certificative fait appel à la démonstration de compétence, la rétroaction et la régulation occupent une place centrale dans l'évaluation formative des apprentissages (Leroux et al., 2019; Nizet et al., 2016).

Les résultats confirment que les deux enseignants utilisent de manière distincte et variable les technologies afin de répliquer, d'amplifier et de transformer les pratiques évaluatives dans un cours en mode hybride et totalement en ligne (Roblyer et Hughes, 2019). Malgré certaines craintes formulées et le manque de repères dans le passage entre la formation en présence et la $F A D$, les résultats révèlent par exemple qu'à défaut d'apporter des transformations radicales au sein des pratiques évaluatives, l'intégration de certaines technologies suscite une plus-value sur le plan de procédés retenus (gain de temps, 
multiplication de la rétroaction, automatisation de la correction). Cela permet de constater que la transformation des pratiques d'évaluation est un processus de négociation complexe impliquant plusieurs éléments et incluant des effets et des possibilités intrinsèques des technologies (Nizet et al., 2016).

Dans une époque où il y a un engouement pour la formation par le numérique, nous pouvons être portés à surestimer l'intégration des technologies comme un moyen pour transformer les pratiques d'évaluation des apprentissages à distance. Or, des enseignants du collégial ont principalement recours au numérique afin de répliquer, d'amplifier et de transformer les évaluations des compétences prévues et stabilisées en amont (Papi, 2016; Roblyer et Hughes, 2019; Leroux et al., 2019). L'intégration judicieuse du TN pour soutenir l'évaluation des apprentissages à distance et la réussite des étudiants est un enjeu crucial (Leroux et al., 2019). La pandémie a bousculé et a mis sous tension les pratiques d'évaluation d'enseignants qui ont dû adapter leurs pratiques évaluatives dans un contexte d'urgence (Detroz, Tessaro et Younès, 2020; Detroz, Malay et Crahay, 2020; Papi et al., 2021; Yerly et Issaieva, 2021). Puisque les changements de pratiques ne prennent de sens que du point de vue des acteurs, les résultats de la recherche apportent une description limitée des transformations, car le phénomène est saisi sous le prisme des pratiques rapportées par deux enseignants recommandés. Pourtant, l'évaluation des apprentissages comprend plusieurs acteurs en FAD du fait de l'intégration des technologies. Malgré ses limites, les résultats de la recherche menée en amont de la pandémie proposent quelques pistes prometteuses qui permettront de répondre à plusieurs questions et à formuler de nouvelles hypothèses de recherche (Papi et al., 2020). Des travaux futurs pourront porter sur un terrain de recherche plus vaste et intégrant plus d'enseignants provenant de différentes disciplines et de programmes à la formation continue et régulière. Cela aurait l'avantage de susciter des nuances et de formuler des exemples riches de pratiques d'évaluation des apprentissages à distance selon le contexte d'enseignement et d'apprentissage, les intentions d'évaluation, les méthodes d'évaluation (les tâches et les instruments d'évaluation), les outils TIC et du Web 2.0, et l'interaction (Leroux et al., 2019). D'autres travaux pourraient examiner les changements entrainés par la transformation des pratiques d'évaluation des apprentissages à distance avec des technologies émergentes comme l'intelligence artificielle (Nolla, 2020).

\section{Liste de références}

Allal, L. (2013). Évaluation : un pont entre enseignement et apprentissage à l'université. Dans M. Romainville, R. Goasdoué, et M. Vantourout (dir.), Évaluation et enseignement supérieur (p. 21- 40). De Boeck Supérieur.

Allen, I. E. et J. Seaman. (2013) Changing Course: Ten Years of Tracking Online Education in the United States, Institute of Education Sciences. https://eric.ed.gov/?id=ED541571

Audet, L. (2011). Les pratiques et défis de l'évaluation en ligne [PDF]. REFAD. http://archives.refad.ca/evaluation en ligne.pdf

Barrette, C. (2009). Métarecherche sur les effets de l'intégration des TIC en pédagogie collégiale. Revue internationale des technologies en pédagogie universitaire / International Journal of Technologies in Higher Education, 6(2-3), 18-25. https://doi.org/10.7202/1000008ar

Basque, J. (2017). L'approche-programme. Les multiples connaissances mobilisées dans un projet d'approche programme en enseignement supérieur. Dans P. Pelletier et A. Huot (dir.), Construire l'expertise pédagogique et curriculaire en enseignement supérieur : Connaissances, compétences et expériences (p. 161-158). Presses de l'Université du Québec. http://extranet.puq.ca/media/produits/documents/3117 9782760548121.pdf

Bélair, K. (2016, 25 avril). La rétroaction audio : un suivi personnalisé. Profweb. https://www.profweb.ca/publications/recits/la-retroaction-audio-un-suivi-personnalise

Bélanger, D.-C., Tremblay, K. et Howe, R. (2012). Portrait actualisé des croyances et des pratiques en évaluation des apprentissages au collégial. Rapport de recherche, Délégation collégiale Performa et Collège de Maisonneuve. 
Bélec, C. et Richard, É. (2019). La rétroaction multitype. Rapport de recherche PAREA. Cégep Gérald Godin et Campus Notre-Dame-De-Foy. https://tinyurl.com/6mtj24sy

Bélisle, M. (2015). Planifier l'évaluation des compétences : une approche globale. Dans J. L. Leroux (dir.), Évaluer les compétences au collégial et à l'université : un guide pratique (p. 130-153). Éditions Chenelière/Association québécoise de pédagogie collégiale (AQPC), Collection Performa.

Benght, M. E., et McDougall, A. G. (2017). NVivo 11 essentials: your guide to the world's most powerful data analysis software. ( $2^{\mathrm{e}}$ édition révisée.). Form \& Kunskap $A B$.

Blais, J.-G., Gilles, J.-L., et Tristan-Lopez, A. (2015). Bienvenue au $21^{e}$ siècle : évaluation des apprentissages et technologies de l'information et de la communication. Dispositif de formation numérique au $1^{\mathrm{er}}$ et $2^{\mathrm{e}}$ cycle universitaire : structuration, outils d'évaluation et perception des étudiants. Peter Lang.

Boettcher, J. V., et Conrad, R.-M. (2016). The Online Teaching Survival Guide: Simple and Practical Pedagogical Tips ( $2^{\mathrm{e}}$ éd.). Jossey-Bass.

Burton, R., Blais, J. G. et Gilles, J.-L. (2013). Technologies et évaluation dans l'enseignement supérieur. Dans M. Romainville, R. Goasdoué, et M. Vantourout, (dir.). Évaluation et enseignement supérieur (p. 85- 105). De Boeck.

Da Costa Cabral, F., Gremion, C., et Roblez, A. (2020). Évaluation formatrice, rythmes et travail de groupes à distance. Journal international de recherche en éducation et formation, numéro hors-série, 1, 77-84.

De Grâce, N. (2016). iSCORE : un portfolio numérique en musique pour le bénéfice des étudiants. Profweb. https://tinyurl.com/4pyshsu2

Detroz, P., Malay, L., et Crahay, V. (2020). Une démarche structurée pour définir quelques conseils en vue de limiter l'impact de la pandémie sur l'évaluation de nos étudiants. Évaluer. Journal international de recherche en éducation et formation. numéro hors-série, 1, 97-110. https://journal.admee.org/index.php/ejiref/article/view/223

Detroz, P., Tessaro, W., et Younès N. (2020). Edito : Évaluer en temps de pandémie. Évaluer. Journal international de recherche en éducation et formation, numéro hors-série, 1, 1-3. https://journal.admee.org/index.php/ejiref/article/view/212

Facchin, S. (2018). La rétroaction traditionnelle ou technologique? Impact du moyen de diffusion de la rétroaction sur la persévérance et la réussite scolaires (rapport de recherche PAREA n PA-2015-024). Montréal, Québec : Cégep à distance.

Fortin, M.-F. et Gagnon, J. (2015). Fondements et étapes du processus de recherche. Méthodes quantitatives et qualitatives $\left(3^{\mathrm{e}}\right.$ éd.). Chenelière Éducation.

Gouvernement du Québec. (2015). La formation à distance dans les universités québécoises : un potentiel à optimiser. Québec : Conseil supérieur de l'éducation. https://www.cse.gouv.qc.ca/fichiers/documents/publications/Avis/50-0486.pd

Gouvernement du Québec. (2018). Stratégie numérique du Québec. Plan d'action numérique en éducation et en enseignement supérieur. Ministère de l'Éducation et de l'Enseignement supérieur. http://www.education.gouv.qc.ca/dossiers-thematiques/plan-daction-numerique/

Gouvernement du Québec. (2019). Cadre de référence de la compétence numérique. Guide pédagogique. Ministère de l'Éducation et de l'Enseignement supérieur. http://www.education.gouv.qc.ca/fileadmin/site web/documents/ministere/Cadre-reference-competence-num.pdf

Gremion, C. (2018). L'évaluation : rôle de synchroniseur des multiples temporalités dans les formations en régime numérique? «Éditorial », Distances et médiations des savoirs, 2. https://doi.org/10.4000/dms.2193

Hughes, J. E. (2000). Teaching English with technology: Exploring teacher learning and practice [thèse de doctorat inédite, Michigan State University, East Lansing, MI]. http://techedges.org/wp-content/uploads/2015/11/Hughes_Full_Dissertation.pdf

Hughes, J. E. (2005). The role of teacher knowledge and learning experiences in forming technology-integrated pedagogy. Journal of Technology and Teacher Education, 13(2), 277-302. http://browningmedportfolio.weebly.com/uploads/2/3/8/1/23811553/hughes 2005 tech-integrated pedagogy.pdf

Johnson, N. (2020). Evolving Definitions in Digital Learning: A national Framework for Categorising Commonly Used Terms. Canadian Digital Learning Research Association. http://www.cdlra-acrfl.ca/wp-content/uploads/2021/07/2021-CDLRA-definitions-report-5.pdf 
Johnson, N., Seamam, J. et Valetsianos, G. (2020). Digital Learning in Canadian Higher Education. Canadian Digital Learning Research Association. http://www.cdlra-acrfl.ca/wp-content/uploads/2021/05/2020 national en.pdf

Jonsson, A. (2014). Rubrics as a Way of Providing Transparency in Assessment. Assessment \& Evaluation in Higher Education, 1-13. https://doi.org/10.1080/02602938.2013.875117

Josephsen, J. (2012). Electronic Portfolios for Distance Learning: A Case from a Nursing Clinical Course. International Journal of ePortfolio, 2(1), 15-27. https://www.theijep.com/articleView.cfm?id=51

Ko, S. et Rossen, S. (2017). Teaching Online: A Practical Guide (4th edition). New York (NY): Routledge.

Koehler, M.J., Mishra, P., Yahya, K. et Yadav, A. (2004). Successful Teaching with Technology: The Complex Interplay of Content, Pedagogy, and Technology. Rapport présenté à la Society for Information Technology \& Teacher Education International Conference (SITE), Atlanta, GA.

Kozanitis, A. (2021). Évaluation des apprentissages en formation à distance : les situations authentiques à la rescousse. Revue internationale des technologies en pédagogie universitaire, 18(1), 276-290. https://doi.org/10.18162/ritpu-2021-v18n1-24

Lakhal, S., Leroux, J. L., et Martel, C. (2015). L'évaluation des apprentissages intégrant les TIC. Dans J. L. Leroux (dir.), Évaluer les compétences au collégial et à l'université : un guide pratique (p. 541-576). Éditions Chenelière/Association québécoise de pédagogie collégiale (AQPC), Collection Performa.

Lebrun, M. (2015). L'hybridation dans l'enseignement supérieur : vers une nouvelle culture de l'évaluation? Évaluer. Journal international de recherche en éducation et formation, 1(1), 65-78. https://journal.admee.org/index.php/ejiref/article/view/93/51

Leduc, D., Raîche, G., et Blais, J.G. (2012). Intégration des pratiques d'évaluation des apprentissages aux pratiques pédagogiques dans le domaine des arts au collégial. Revue des sciences de l'éducation, 38(2), 373-396. https://doi.org/10.7202/1019611ar

Leroux, J. L. (2018, 29 mai). L'évaluation des apprentissages à distance dans un programme en approche par compétences. Profweb : multidisciplinaire. https://tinyurl.com/2p925e9n

Leroux, J. L. (2010). Analyse des pratiques évaluatives d'enseignantes et d'enseignants dans une approche par compétences au collégial [thèse de doctorat]. http://savoirs.usherbrooke.ca/handle/11143/947

Leroux, J. L. (2016). Le jugement évaluatif d'enseignants du supérieur dans un contexte d'évaluation de compétences. Dans L. Mottier Lopez et W. Tessaro (dir.), Le jugement professionnel au cœur de l'évaluation et de la régulation des apprentissages (p. 169-193). Peter Lang.

Leroux, J. L. et Bélair, L. (2015). Exercer son jugement professionnel en enseignement supérieur. Dans J. L. Leroux (dir.), Évaluer les compétences au collégial et à l'université : un guide pratique (p. 67-107). Éditions Chenelière/AQPC, Collection Performa.

Leroux, J. L., Boyer, M., Corriveau, L. et Nolla, J.-M. (2017, juin). Processus d'évaluation des compétences en formation à distance dans une approche collaborative en enseignement supérieur. Actes du IX colloque : Questions de pédagogies dans l'enseignement supérieur (QPES) : Relever les défis de l'Altérité dans l'enseignement supérieur (p. 377-385). Grenoble, France.

Leroux, J. L., Desrochers, M.-Ė, et Myre-Bourgault, M. (2019). L'évaluation des apprentissages à l'ère du numérique en enseignement supérieur : quels besoins et quels défis? Évaluer. Journal international de recherche en éducation et formation, 5 (3), 85-108. https://journal.admee.org/index.php/ejiref/article/view/209

Liang, X. et Creasy, K. (2004). Classroom assessment in Web-based instructional environment: instructors' experience. Practical Assessment, Research and Evaluation, 9(7). https://files.eric.ed.gov/fulltext/ED494681.pdf

Lison, C., Bédard, D., Beaucher, C. et Trudelle, D. (2014). De l'innovation à un modèle dynamique innovationnelle en enseignement supérieur. Revue internationale de pédagogie de l'enseignement supérieur, 30(1). https://doi.org/10.4000/ripes.771

Louis, R., et Bédard, D. (2015). Les tendances en évaluation des apprentissages en enseignement supérieur. Dans J. L. Leroux (dir.), Évaluer les compétences au collégial et à l'université : un guide pratique (p. 23-64). Montréal, Québec : Éditions Chenelière/ AQPC, Collection PERFORMA.

Meyer, F., et Lakhal, S. (2017). Qu'est-ce que le Blended learning? Communication présentée dans le cadre de la CIRTA. https://www.cirta.org/index.php/features/blog/36-accueil/258-webinaire-qu-est-ce-que-le-blended-learning. 
Miles, M. B. et Huberman, A. M. (2003). Analyse des données qualitatives. Paris, France : De Boeck Université.

Mottier Lopez, L. (2015). Évaluations formative et certificative des apprentissages : enjeux pour l'enseignement. De Boeck.

Nizet, I., Leroux, J. L., Deaudelin, C., Béland, S., et Goulet, J. (2016). Bilan de pratiques évaluatives à distance en contexte de formation universitaire. Revue internationale de pédagogie de l'enseignement supérieur, 32(2), 1-25. https://doi.org/10.4000/ripes.1073

Nolla, J.-M. (2020). Les changements entrainés par les technologies du numérique (TN) en évaluation des apprentissages en formation à distance (FAD) : l'adaptation des professeures et professeurs en éducation. tThèse de doctorat inédite, Université de Sherbrooke]. https://savoirs.usherbrooke.ca/handle/11143/17131

Papi, C. (2016). De l'évolution du métier d'enseignant à distance. STICEF, 23. https://r-libre.teluq.ca/920/1/sticef_2016_NS_papi_03p.pdf

Papi, C., Brassard, C., Plante, P., Savard, I., Mendoza, G. A. et Gérin-Lajoie, S. (2021). Créer dans l'urgence une formation à distance de qualité pour former... à la formation à distance : tout un défi! Revue internationale des technologies en pédagogie universitaire, 18(1), 233-240. https://doi.org/10.18162/ritpu-2021-v18n1-20

Papi, C., Gérin-Lajoie, S. et Hébert, M.-H. (2020). Se rapprocher de l'évaluation à distance : Dix pistes de réponse. Évaluer. Journal international de recherche en éducation et formation, numéro hors-série 1, 201-206. https://journal.admee.org/index.php/ejiref/article/view/233

Petit, M. (2016). Caractéristiques d'une supervision à distance de stagiaires en enseignement en ce qui concerne la création d'un sentiment de présence. Revue internationale de pédagogie de l'enseignement supérieur, 32-1. https://doi.org/10.4000/ripes.1041

Prégent, R., Bernard, H., et Kozanitis, A. (2009). Enseigner à l'université dans une approche-programme : guide à l'intention des nouveaux professeurs et chargés de cours. Presses internationales Polytechnique.

Roblyer, M. D. et Hughes, J. E. (2019). Integrating Educational Technology into Teaching. Transforming Learning Across, Disciplines ( $8^{\mathrm{e}}$ éd.). Boston, MA : Pearson.

Roy, N., Gruslin, É., et Poelhuber, B. (2020). Le plan d'action du numérique en éducation et en enseignement supérieur au Québec : mesures et initiatives au collégial. Revue internationale des technologies en pédagogie universitaire, 17-1, p. 63-75. https://doi.org/10.18162/ritpu-2020-v17n1-13

Savoie-Zajc, L. (2004). L'entrevue semi-dirigée. Dans B. Gauthier (dir.), Recherche sociale. De la problématique à la collecte des données (p. 293-316). Presses de l'Université du Québec.

Savoie-Zajc, L. (2011). La recherche qualitative/interprétative en éducation. Dans T. Karsenti et L. Savoie-Zajc (dir.), La recherche en éducation : étapes et approches ( $3^{e}$ éd.) (p. 123-148). Éditions du Renouveau pédagogique inc.

Scallon, G. (2004). L'évaluation des apprentissages dans une approche par compétences. Éditions du renouveau pédagogique inc.

Seaman, J.E., Allen, I.E., et Seaman, J. (2018) Grade Increase: Tracking Distance Education in the United States. Wellesley MA: The Babson Survey Research Group. https://eric.ed.gov/?id=ED580852

Stein, J., et Graham, C. R. (2014). Essentials for blended learning: A standards-based guide. Routledge.

Stödberg, U. (2012). A research review of e-assessment. Assessment \& Evaluation in Higher Education, 37(5), 591-604. https://doi.org/10.1080/02602938.2011.557496

Tardif, J. (2006). L'évaluation des compétences. Documenter le parcours de développement. Chenelière Éducation.

Tremblay, B. (2020). Le numérique, plus que jamais au service de la mission d'enseignement des cégeps. Revue internationale des technologies en pédagogie universitaire/International Journal of Technologies in Higher Education, 17(1), 15-17. https://doi.org/10.18162/ritpu-2020-v17n1-05

UNESCO (2011). TIC UNESCO : un référentiel de compétences pour les enseignants. UNESCO.

Vai, M., et Sosulski, K. (2015). Essentials of Online Course Design: A Standards-Based Guide (2 ${ }^{\mathrm{e}}$ éd.). Routledge.

Van Der Maren, J.-M. (1996). Méthodes de recherche pour l'éducation ( $2^{\mathrm{e}}$ éd.). Presses de l'Université de Montréal (ouvrage original publié en 1995).

Yerly, G., Issaieva, E. (2021). (Re)penser l'évaluation des apprentissages au postsecondaire en temps de crise : défis à relever et occasions à saisir en période de COVID-19. Revue internationale des technologies en pédagogie universitaire,18(1), 89-101. https://doi.org/10.18162/ritpu-2021-v18n1-09 